\title{
Tumour necrosis is a postoperative prognostic marker for pancreatic cancer patients with a high interobserver reproducibility in histological evaluation
}

\author{
N Hiraoka*,', Y Ino', S Sekine', H Tsuda ${ }^{2}$, K Shimada ${ }^{3}$, T Kosuge ${ }^{3}$, J Zavada $^{4}$, M Yoshida ${ }^{2}$, K Yamada ${ }^{2}$, \\ T Koyama ${ }^{2}$ and $Y$ Kanai'
}

'Pathology Division, National Cancer Center Research Institute, 5-I-I Tsukiji, Chuo-ku, Tokyo 104-0045, Japan; ${ }^{2}$ Clinical Laboratory Division, National Cancer Center Hospital, 5-I - I Tsukjij, Chuo-ku, Tokyo I 04-0045, Japan; ${ }^{3}$ Hepato-Biliary and Pancreatic Surgery Division, National Cancer Center Hospital, 5-I-I Tsukiji, Chuo-ku, Tokyo 104-0045, Japan; ${ }^{4}$ Institute of Organic Chemistry and Biochemistry, Flemingovo nam.2, I 66 I0 Prague 6, Czech Republic

BACKGROUND: Tumour necrosis reflects the presence of hypoxia, which can be indicative of an aggressive tumour phenotype. The aim of this study was to investigate whether histological necrosis is a useful predictor of outcome in patients with pancreatic ductal carcinoma (PDC).

METHODS: We reviewed histopathological findings in 348 cases of PDC in comparison with clinicopathological information. We counted small necrotic foci (micronecrosis) as necrosis, in addition to massive necrosis that had been only defined as necrosis in previous studies. The reproducibility of identifying histological parameters was tested by asking five independent observers to blindly review 5 I examples of PDC.

RESULTS: Both micronecrosis and massive necrosis corresponded to hypoxic foci expressing carbonic anhydrase IX detected by immunohistochemistry. Multivariate survival analysis showed that histological necrosis was an independent predictor of poor outcome in terms of both disease-free survival (DFS) and disease-specific survival (DSS) of PDC patients. In addition, metastatic status, and lymphatic, venous, and intrapancreatic neural invasion were independent prognostic factors for shorter DFS and metastatic status, margin status, lymphatic invasion, and intrapancreatic neural invasion were independent prognostic factors for DSS. The interobserver reproducibility of necrosis identification among the five independent observers was 'almost perfect' ( $\kappa$-value of 0.87 ).

CONCLUSION: Histological necrosis is a simple, accurate, and reproducible predictor of postoperative outcome in PDC patients. British Journal of Cancer (2010) 103, 1057-1065. doi:I0.1038/sj.bjc.6605854 www.bjcancer.com

Published online 24 August 2010

(c) 2010 Cancer Research UK

Keywords: necrosis; pancreatic cancer; prognostic factor; interobserver reproducibility; hypoxia

Pancreatic cancer (pancreatic ductal carcinoma (PDC)) is the fourth and fifth leading cause of cancer-related death in the United States and Japan, respectively (Center for Cancer Control and Information Services and National Cancer Center, 2009; Jemal et al, 2009). Because of its aggressive growth and early metastatic dissemination, the overall 5-year survival rate for patients with pancreatic cancer is $3-5 \%$, and that of patients treated by curative resection is $15-25 \%$ (Klöppel et al, 2000; Lim et al, 2003; Hruban et al, 2007; Jemal et al, 2009). The mortality rate has not shown any obvious improvement for decades. The development of predictive biomarkers to assist the selection of patient subsets is useful for studies aimed at reducing the mortality of PDC patients, especially in phase clinical studies evaluating various therapeutic approaches (Philip et al, 2009).

Several histopathological parameters such as tumour size (Yeo et al, 1995; Sohn et al, 2000; Lim et al, 2003; Shimada et al, 2006), tumour histological grade (Luttges et al, 2000; Adsay et al, 2005),

*Correspondence: Dr N Hiraoka; E-mail: nhiraoka@ncc.go.jp Received 24 May 20I0; revised I4 July 20I0; accepted 20 July 20I0; published online 24 August 2010 lymph nodal metastasis (Trede et al, 1990; Lim et al, 2003; Schnelldorfer et al, 2008), and lymphatic, venous (Luttges et al, 2000; Takai et al, 2003), and neural invasion (Mitsunaga et al, 2005, 2007) have been proposed as hallmarks predictive of postoperative outcome in patients with PDC. Stage is the most important prognosticator (Klöppel et al, 2000; Hruban et al, 2007), although majority of resectable cases were classified into advanced stage, stage IIB of the International Union Against Cancer (UICC) tumour-node-metastasis (TNM) classification and were not able to be stratified more precisely. Histopathological evaluation of PDC can be performed using routine diagnostic techniques in pathology departments without any additional equipment or special expertise. The predictive values of the above mentioned parameters are sometimes controversial and complex, and some have problems related to interobserver reproducibility. To avoid these pitfalls and to stratify PDCs in a distinct and objective manner, molecular or genetic markers have been developed, although these often require special equipment or expertise and cannot usually be performed on a routine basis. Therefore, simpler, more reproducible, and easily assessable histopathological predictors are needed. 
Hypoxia is a common feature of human cancers, which induces a transcription programme mediated mainly by hypoxia-inducible factor- $1 \alpha$ (HIF- $1 \alpha)$ that promotes aggressive tumour phenotype (Harris, 2002; Vaupel and Mayer, 2007; Bristow and Hill, 2008). It is a prognostic indicator in many solid tumours (Vaupel and Mayer, 2007), and is often detected by examining the expression of carbonic anhydrase IX (CAIX) (Chia et al, 2001), which is a regulator of cellular $\mathrm{pH}$ and its expression is induced by HIF- $1 \alpha$ (Harris, 2002; Bristow and Hill, 2008). Intratumoural hypoxia is reflected histologically by the presence of necrosis, which has also been reported to be a prognostic factor in patients with breast (Gilchrist et al, 1993) and bladder (Ord et al, 2007) cancers. Hypoxia is evident in PDCs, in which expression of HIF- $1 \alpha$ and CAIX has been detected in $60-70 \%$ and $78 \%$ of cases, respectively (Kitada et al, 2003; Couvelard et al, 2005; Sun et al, 2007), whereas histological necrosis has been found in only $30-40 \%$ of PDCs in previous studies (Couvelard et al, 2005; Mitsunaga et al, 2005). Neither large-scale study nor multivariate survival study has been performed to evaluate prognostic value of hypoxia in PDC patients.

In this study, we found that necrotic areas were present in more than $60 \%$ of PDCs when small necrotic foci were taken into account. We also found that CAIX were expressed in and around these necrotic lesions, indicating that these areas were in a condition of hypoxia. Therefore, detection of necrosis using our definition may offer a chance of stratifying PDC patients for tissue hypoxia more accurately. In this study, with the aim of investigating whether histologically evident necrosis is useful for prediction of patient outcome among various histopathological parameters, and we reviewed histopathological findings in 348 cases of PDC in comparison with clinicopathological information.

\section{MATERIALS AND METHODS}

\section{Study population}

This study was approved by the Ethics Committee of the National Cancer Center, Japan. Clinical and pathological data were obtained through a detailed retrospective review of the medical records of all 348 patients with ductal carcinoma of the pancreas that had undergone initial surgical resection between 1990 and 2005 at the National Cancer Center Hospital. None of the patients had received any previous therapy. All patients received standard therapy appropriate for their clinical stages. The operative procedures included 228 pancreatoduodenectomies or pylorus-preserving pancreatoduodenectomies, 104 distal pancreatectomies, and 16 total pancreatectomies. Along with tumour extension, lymphadenectomy was performed at the hepatoduodenal ligament and around the abdominal aorta. All the patients included in this study underwent macroscopic curative resection, which was defined as the macroscopic removal of all gross tumours without liver metastases, macroscopic peritoneal dissemination, bulky lymph node involvement, or apparent tumour invasion around the common hepatic or superior mesenteric arteries after routine examination using intraoperative ultrasonography. All of the cases were conventional ductal carcinomas and adenocarcinomas originating in intraductal papillary mucinous neoplasms or mucinous cystic neoplasms were excluded. Secondary tumours and postneoadjuvant cases were also excluded. The clinicopathological characters of the patients were summarized in Table 1. All M1 patients had nodal metastasis around the abdominal aorta, without any other form of metastasis. Males accounted for 206 patients and females for 142; the mean patient age was 62.9 years (range, 27-87 years). Every patient was followed up in the outpatient clinic every 1-3 month during the first postoperative year, and every 6-12 months thereafter. Patients underwent physical examination, laboratory tests, chest radiography, abdominal computed tomography, and/or ultrasonography, unless there was a confirmed relapse. The tumour markers carcinoembryonic antigen and carbohydrate antigen 19-9 were also measured until relapse. Recurrence was suspected when a new local or distant metastatic lesion was found on serial images and an increase in tumour marker levels was recognised. When progression of the disease was confirmed by repeated imaging studies, the date of the first suspicious radiologic finding was used as the date of initial disease recurrence. The median follow-up period after surgery was $17.9(1.3-210)$ months for the patients overall: 69 patients $(19.8 \%)$ were alive at the census date (June 2009), $239(68.7 \%)$ died because of pancreatic cancer, and $40(11.5 \%)$ died of other causes. Postresection adjuvant therapy information was available for 327 patients, of whom 19 received chemotherapy and radiotherapy, 134 received chemotherapy only, 2 received radiotherapy only, and 172 did not receive any additional therapy.

\section{Pathological examination}

All of the ductal carcinomas were pathologically reexamined and were classified according to the World Health Organisation classification (Klöppel et al, 2000), UICC TNM classification (Wittekind et al, 2005), and the Classification of Pancreatic Carcinoma of the Japan Pancreas Society (JPS) (Japan Pancreas Society, 2003). Surgically resected specimens were fixed in $10 \%$ formalin and cut into serial 5-mm-thick slices, horizontally in the pancreas head, and sagittally in the pancreas body and tail. All the sections were stained with hematoxylin and eosin for pathological examination. The following histopathological variables were evaluated according to the classification of JPS: tumour histological grade, nerve plexus invasion, and lymphatic, venous, and intrapancreatic neural invasion.

Tumour necrosis in PDCs was reported previously (Couvelard et al, 2005; Mitsunaga et al, 2005), having been defined as 'confluent cell death in invasive areas of primary cancer, visible at an objective lens magnification of $\times 4^{\prime}$ (Mitsunaga et al, 2005), which is the same to the definition having been mentioned in breast cancer. Tumour histology often varies among the organs in which tumours develop. We noticed that small areas of necrosis were evident in PDCs, wherein gland formation by cancer cells was ruptured, usually in association with neutrophil infiltration. We refer to this hereafter as micronecrosis, and to the former as massive necrosis. As sometimes it is difficult to differentiate necrotic lesions into either of these two patterns, we combined these two types of lesions solely as 'necrosis'. The definition of histological necrosis is as follows. Necrosis occurs in cancer tissue regardless of its extent, and is usually found in both cancer cells and cancer stroma (Figure 1). When coagulation necrosis is extensively developed (massive necrosis), it corresponds to what was referred to as necrosis previously (Couvelard et al, 2005; Mitsunaga et al, 2005). Smaller areas of necrosis (micronecrosis) often recognised adjacent to ruptured cancer-forming tubules is almost always accompanied by neutrophil infiltration (Figure 1).

\section{Interobserver reproducibility in identifying histological characteristics}

To test the reproducibility of identification of histological characteristics, five independent observers (SS, HT, MY, KY, and TK) were asked to review 51 examples of IDC that were consecutive cases surgically resected between 1997 and 2000. One slide was selected from each of the 51 cases. This slide was one of the slides containing the maximum cut section of the tumour. The complete set of slides from the 51 cases was sent to the five independent observers, all of whom are general surgical pathologists with no specific expertise in the pancreas, and who encounter pancreas specimens rarely. One observer (HT) specialises in breast and female genitourinary pathology and one (SS) in 
Table I Relationship between clinicopathological characteristics and histological necrosis

\begin{tabular}{|c|c|c|c|c|}
\hline \multirow[b]{2}{*}{ Characteristics } & \multirow[b]{2}{*}{ No. of patients } & \multicolumn{2}{|c|}{ Necrosis } & \multirow[b]{2}{*}{$P$} \\
\hline & & Presence & Absence & \\
\hline \multicolumn{5}{|l|}{ Age, years } \\
\hline$<60$ & 123 & 75 & 48 & \\
\hline$\geqslant 60$ & 225 & 148 & 77 & 0.414 \\
\hline \multicolumn{5}{|l|}{ Sex } \\
\hline Male & 206 & 143 & 63 & \\
\hline Female & 142 & 80 & 62 & 0.017 \\
\hline \multicolumn{5}{|l|}{ Localisation } \\
\hline Pancreas head & 228 & 147 & 81 & \\
\hline Pancreas body or tail & 109 & 70 & 39 & 1.000 \\
\hline \multicolumn{5}{|l|}{ Size $(m m)$} \\
\hline$<30$ & 83 & 42 & 41 & \\
\hline$\geqslant 30$ & 265 & $|8|$ & 84 & 0.004 \\
\hline \multicolumn{5}{|l|}{ Pathologic tumour status } \\
\hline $\mathrm{Tl}$ & 6 & 1 & 5 & \\
\hline $\mathrm{T} 2$ & 3 & 3 & 0 & \\
\hline T3 & 339 & 219 & 120 & \\
\hline $\mathrm{T} 4$ & 0 & 0 & 0 & $0.023^{a}$ \\
\hline \multicolumn{5}{|l|}{ Pathologic node status } \\
\hline No & 64 & 33 & 31 & \\
\hline $\mathrm{NI}$ & 284 & 190 & 94 & 0.030 \\
\hline \multicolumn{5}{|c|}{ Pathologic metastasis status } \\
\hline Mo & 310 & 193 & 117 & \\
\hline MI & 38 & 30 & 8 & 0.049 \\
\hline \multicolumn{5}{|l|}{ Stage } \\
\hline IA & 3 & 0 & 3 & \\
\hline IB & 2 & 2 & 0 & \\
\hline$\| \mathrm{A}$ & 59 & 31 & 28 & \\
\hline$\| B$ & 246 & 160 & 86 & \\
\hline III & 0 & 0 & 0 & \\
\hline IV & 38 & 30 & 8 & $0.009^{a}$ \\
\hline \multicolumn{5}{|l|}{ Tumour histological grade } \\
\hline W/D & 90 & 41 & 49 & \\
\hline M/D & 181 & 122 & 59 & \\
\hline P/D & 77 & 60 & 17 & $<0.000 \mathrm{I}^{\mathrm{a}}$ \\
\hline \multicolumn{5}{|l|}{ Tumour margin status } \\
\hline Negative & 249 & 166 & 82 & \\
\hline Positive & 100 & 57 & 43 & 0.085 \\
\hline \multicolumn{5}{|l|}{ Nerve plexus invasion ${ }^{\mathrm{b}}$} \\
\hline Absence & 112 & 65 & 47 & \\
\hline Presence & 236 & 158 & 78 & 0.120 \\
\hline \multicolumn{5}{|l|}{ Lymphatic invasion $^{\mathrm{b}}$} \\
\hline 0,1 & 102 & 58 & 44 & \\
\hline 2,3 & 246 & 165 & 81 & 0.086 \\
\hline \multicolumn{5}{|l|}{ Venous invasion ${ }^{\mathrm{b}}$} \\
\hline 0,1 & 124 & 63 & 61 & \\
\hline 2,3 & 224 & 160 & 64 & 0.0002 \\
\hline \multicolumn{5}{|c|}{ Intrapancreatic neural invasion ${ }^{\mathrm{b}}$} \\
\hline 0,1 & $|4|$ & 86 & 55 & \\
\hline 2,3 & 207 & 137 & 70 & 0.363 \\
\hline \multicolumn{5}{|l|}{ Recurrent site $^{c}$} \\
\hline Local & 54 & 39 & 15 & \\
\hline Distant sites & 198 & 134 & 64 & 0.620 \\
\hline
\end{tabular}

Table I (Continued) gastrointestinal tract pathology. These observers were provided with the definition of 'histological necrosis' according to Figure 1 and with the definitions for lymphatic, venous, and neural invasion stated in the classification of JPS (Japan Pancreas Society, 2003). They were asked to assess the presence of histological necrosis and each of the grades of lymphatic, venous, and neural invasion evident within each of the provided slides. These observers were blind to the identity of the original reviewers or those of each other. They were also not provided with any clinical information on the outcome of the patients.

\section{Immunohistochemical analysis}

Immunohistochemistry was performed on formalin-fixed, paraffin-embedded tissue sections as described previously (Takahashi et al, 2007), using antibodies against CAIX (M75, 1:200) (Pastorekova et al, 1992; Zavada et al, 2000) and HIF-1 $\alpha$ (54, $1: 500$, BD Transduction Laboratories, Franklin Lakes, NJ, USA). Avidin-biotin complex method and CSA system (DAKO, Glostrup, Denmark) were used for these immunohistochemistry, respectively. The sections were autoclaved in the buffer ( $\mathrm{pH} 9.0$, Nichirei Biosciences, Tokyo, Japan) for antigen retrieval. For immunohistochemical examination of CAIX in PDCs, we used sections of representative blocks from 203 cases of PDC. Carbonic anhydrase IX is expressed always in the crypt enterocytes of the duodenum and sometimes in normal epithelial cells of the pancreatic duct and pancreatic intraepithelial neoplasm. These cells were used as the positive control for CAIX immunohistochemistry. Immunohistochemistry was performed without primary antibody for negative control. When more than $20 \%$ of cancer cells in the specimen expressed CAIX, the case was judged as positive for CAIX in cancer cells. When there were any stromal cells expressing CAIX in cancer tissue, the case was judged as presence of stromal cells expressing CAIX.

\section{Statistical analysis}

Comparisons of qualitative variables were performed using the $\chi^{2}$ test or Fisher's exact test. One-way analysis of variance was used to compare the means of three or more groups. The postoperative disease-free survival (DFS) and disease-specific survival (DSS) rates were calculated by the Kaplan-Meier method. Univariate analysis was performed for prognostic factors using the log-rank test. The factors found to be predictive by univariate analysis were subjected to multivariate analysis using the Cox proportional hazards model (backward elimination method). Interobserver 

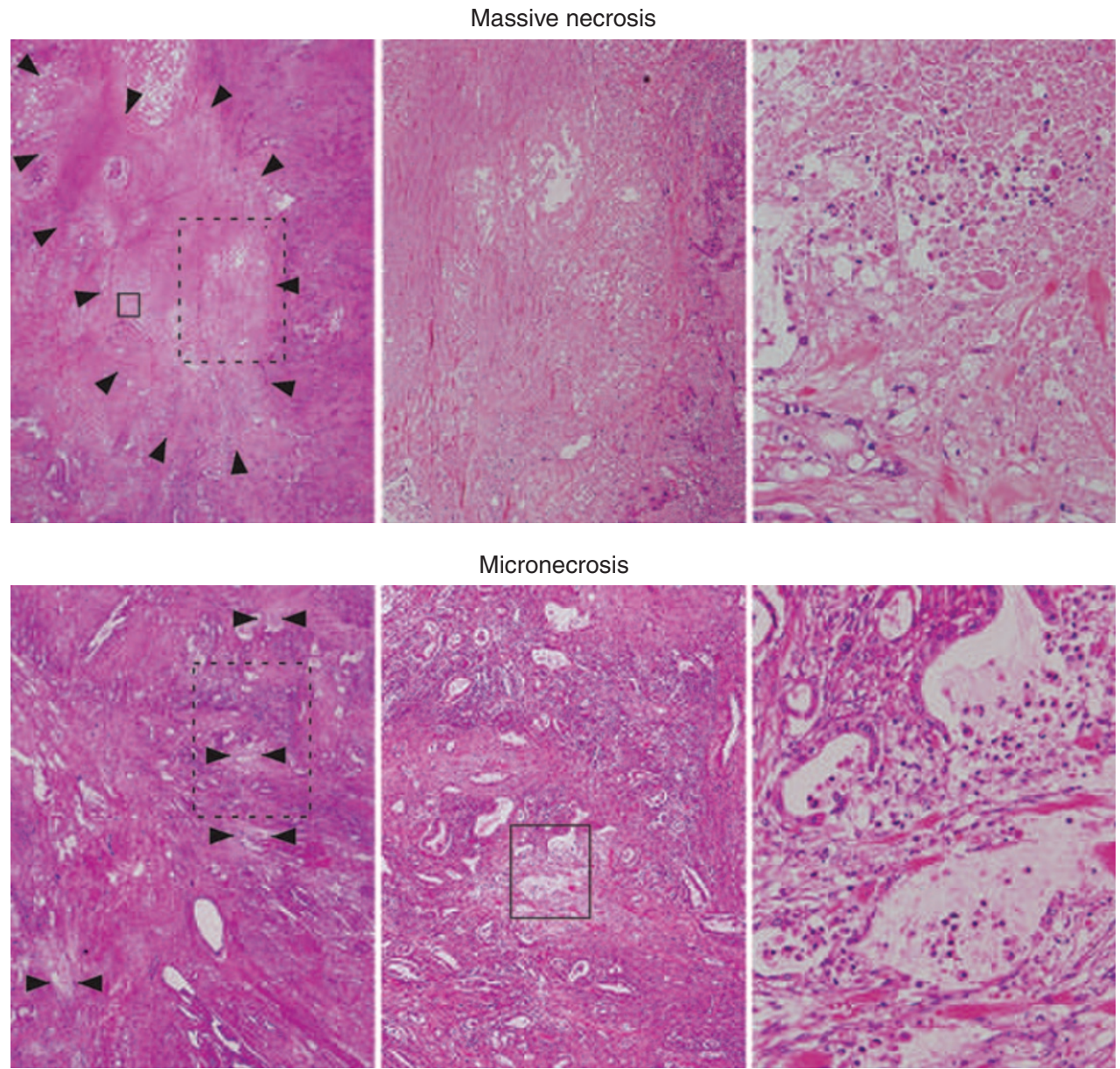

Figure I Representative histology of massive necrosis (upper columns) and micronecrosis (lower columns). Arrows indicate necrotic area. Left, centre, and right columns are in low $(\times 6.25)$, middle $(\times 20)$, and high magnification $(\times 100)$, respectively. High power view of histology in right columns corresponds to the rectangle (solid line) in left or middle column. Middle power view of histology in centre columns corresponds to the rectangle (dotted line) in left columns.

agreement (reproducibility) was tested by obtaining the $\kappa$-scores (Fleiss, 1971; Landis and Koch, 1977). Differences at $P<0.05$ were considered statistically significant. Statistical analyses were performed with StatView-J 5.0 software (Abacus Concepts, Berkeley, CA, USA).

\section{RESULTS}

\section{Correspondence of both massive necrosis and micronecrosis with hypoxic foci}

Immunohistochemical analysis revealed that CAIX was expressed in cancer cells or stromal cells within or around areas of both massive necrosis and micronecrosis (Figure 2).

\section{Prognostic significance of the presence of hypoxic foci detected by expression of CAIX in cancer stromal cells}

Survival analysis showed that the presence of hypoxic foci with expression of CAIX in stromal cells in cancer tissue was closely associated with shorter DFS $(P=0.004)$ and DSS $(P=0.003)$ (Figure 3 ). The presence of cancer cells expressing CAIX was also associated with shorter survival rates (Figure 3), although its occasional expression in cancer cells forming well-differentiated glands distant from necrotic areas probably indicated a cellular phenotype similar to that of normal ductal epithelial cells, and was unrelated to hypoxia. Then we used expression of CAIX in cancer stromal cells as hypoxic marker in this study. Multivariate Cox regression analysis revealed that the presence of hypoxic foci was an independent predictor of shorter DFS $(P=0.005)$ and DSS $(P=0.011)$ (Supplementary Table 1$)$. The presence of necrosis was significantly correlated with the presence of hypoxic foci (Table 1). More CAIX-expressing cells were found in larger areas of necrosis.

\section{Histopathological evaluation of PDC}

Table 1 lists the clinicopathological features of patients with PDC. When correlations with these clinicopathological features were analyzed, the presence of necrosis was found to be more likely in cases with large tumours $(P=0.004)$, higher tumour status $(P=0.023)$, presence of nodal metastasis $(P=0.030)$, presence of distant metastasis $(P=0.049)$, higher TNM stage $(P=0.009)$, poorer tumour differentiation $(P<0.0001)$, and more frequent venous invasion $(P=0.0002)$.

\section{Prognostic significance of the histopathological valuables}

Survival analysis demonstrated an association between the presence of necrosis and shorter DFS $(P<0.0001 ; \mathrm{HR}=2.007$; 95\% CI: $1.531-2.630)$ and DSS $(P<0.0001$; HR $=2.196 ; 95 \% \mathrm{CI}$ : 

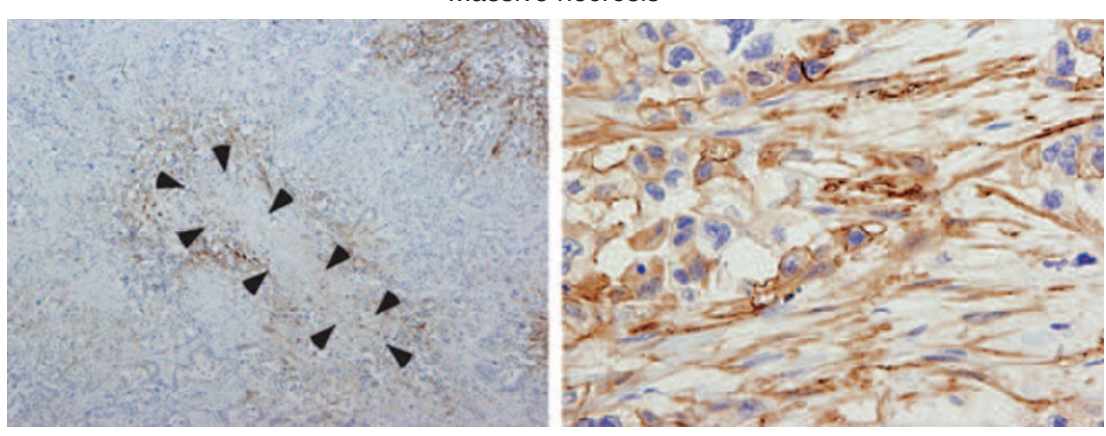

Micronecrosis
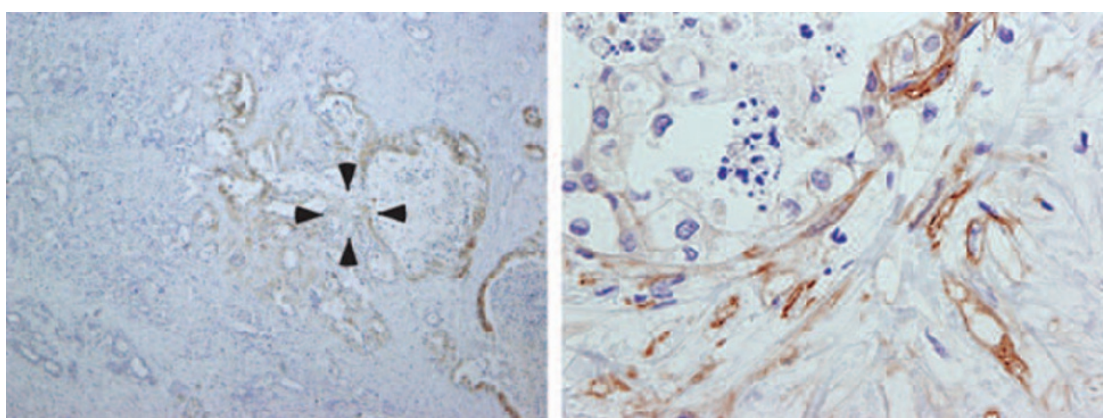

Figure 2 Hypoxia is reflected by the presence of massive necrosis or micronecrosis. Expression of CAIX is immunohistochemically detectable in both cancer cells and stromal cells within or around areas of massive necrosis (upper columns) and micronecrosis (lower columns). Carbonic anhydrase IX is expressed in plasma membrane. Arrows indicate necrotic area. Low power view (left columns) and high power view (right columns).
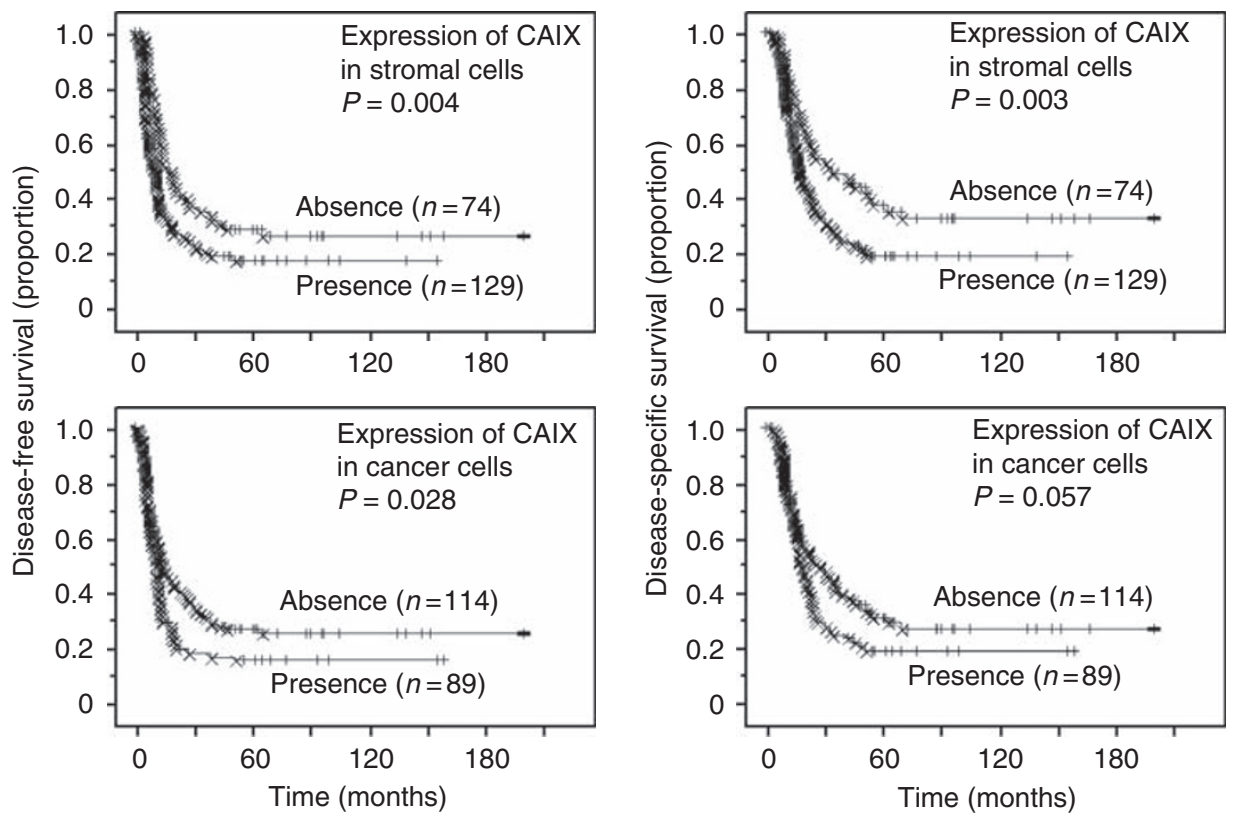

Figure 3 Kaplan-Meier survival curves showing the comparison of disease-free survival between high and low expression of CAIX ( $P$-values obtained from log-rank test) (left columns). Kaplan-Meier survival curves showing the comparison of disease-specific survival between high and low expression of CAIX ( $P$-values obtained from log-rank test) (right columns).

1.659-2.905) (Figure 4). A similar association was found in patients with PDC at each TNM stages (Figure 4).

The average survival periods for patients having PDC with and without necrosis were $24.62 \pm 1.42$ months and $47.36 \pm 2.75$ months, respectively. One-year survival rates for patients having
PDC with and without necrosis were $63.3 \pm 3.3 \%$ and $89.1 \pm 2.9 \%$, respectively; the 2-year rates were $36.2 \pm 3.4 \%$ and $69.0 \pm 4.3 \%$, and the 5 -year rates were $17.1 \pm 2.9 \%$ and $40.1 \pm 4.8 \%$.

Multivariate Cox regression analysis showed that necrosis $(P<0.0001 ; \mathrm{HR}=1.853 ; 95 \% \mathrm{CI}: 1.407-2.440)$, metastatic status, 

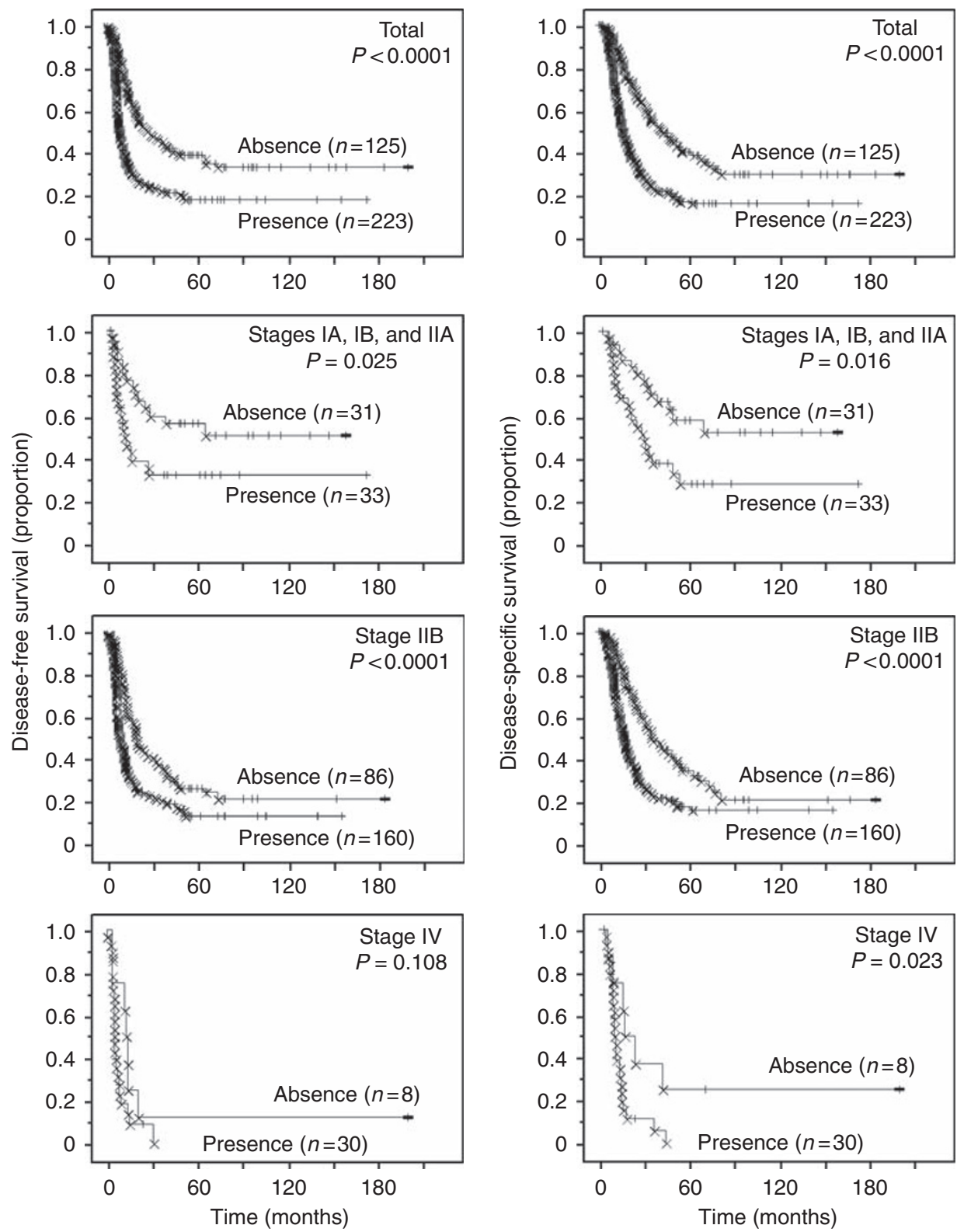

Figure 4 Kaplan-Meier survival curves showing a comparison of disease-free survival between cases in which histological necrosis was present and absent ( $P$-values obtained by log-rank test) (left columns). Kaplan-Meier survival curves showing a comparison of disease-specific survival between cases, in which histological necrosis was present and absent ( $P$-values obtained by log-rank test) (right columns).

lymphatic invasion, venous invasion, and intrapancreatic neural invasion were independent predictors of DFS, and that necrosis $(P<0.0001 ; \mathrm{HR}=2.238 ; 95 \% \mathrm{CI}: 1.686-2.971)$, metastatic status, margin status, lymphatic invasion, and intrapancreatic neural invasion were independent predictors of DSS (Table 2).

When massive necrosis and micronecrosis were separated as distinct variables, univariate survival analysis demonstrated an association between the presence of massive necrosis and shorter DFS $(P<0.0001)$ and DSS $(P<0.0001)$, and between the presence of micronecrosis and shorter DFS $(P=0.004)$ and DSS $(P=0.001)$ (Supplementary Table 2). Multivariate Cox regression analysis showed that massive necrosis $(P=0.0006)$ and micronecrosis $(P=0.0004)$ were independent predictors of shorter DFS and that massive necrosis $(P<0.0001)$ and micronecrosis $(P<0.0001)$ were independent predictors of shorter DSS (Supplementary Table 2).

\section{Reproducibility of necrosis identification by independent observers}

Interobserver agreement (reproducibility) regarding the identification of necrosis among the five independent observers who reviewed the 51 slides blindly had a $\kappa$-value of 0.87 . On the other hand, the corresponding $\kappa$-values for grading $(0-3)$ of lymphatic, venous, and neural invasion were $0.11,0.11$, and 0.31 , respectively. According to the widely used statistical chart that grades the strength of agreement (Fleiss, 1971; Landis and Koch, 1977) into 6 categories (poor ( $\kappa$-value, $<0.00)$, slight $(0.00-0.20)$, fair $(0.21-0.40)$, moderate $(0.41-0.60)$, substantial $(0.61-0.80)$, and almost perfect $(0.81-1.00))$, the agreement for identification of necrosis and the grading for lymphatic, venous, and neural invasion were categorized as 'almost perfect', 'slight', 'slight', and 'fair', respectively. When the grades for lymphatic, venous, and neural 
Table 2 Univariate and multivariate analyses of prognostic factors associated with disease-free survival in patients with ductal carcinoma of the pancreas $(n=348)$

\begin{tabular}{|c|c|c|c|c|}
\hline Variables & \multicolumn{2}{|c|}{ Univariate analysis } & \multicolumn{2}{|c|}{ Multivariate analysis } \\
\hline Gender (male/female) & $0.987(0.764-1.274)$ & 0.919 & & \\
\hline Localisation (pancreas head/body or tail) & $0.865(0.657-1.140)$ & 0.304 & & \\
\hline Tumour size $(\geqslant 30 \mathrm{~mm} /<30 \mathrm{~mm})$ & $1.789(1.306-2.450)$ & 0.0003 & & \\
\hline Pathologic tumour status $(\mathrm{TI}+\mathrm{T} 2 / \mathrm{T} 3)$ & $3.863(1.235-12.1)$ & 0.020 & & \\
\hline Histological grade (W/D/M/D, P/D) ${ }^{a}$ & $1.507(1.122-2.025)$ & 0.006 & & \\
\hline Tumour margin status (negative/positive) & $1.470(1.121-1.929)$ & 0.005 & & \\
\hline PL (absence/presence) ${ }^{\mathrm{a}}$ & $1.560(1.182-2.060)$ & 0.002 & & \\
\hline Lymphatic invasion $(0,1 / 2,3)^{\mathrm{a}}$ & $2.040(1.513-2.75 \mid)$ & $<0.0001$ & $1.475(1.068-2.038)$ & 0.018 \\
\hline Venous invasion $(0,1 / 2,3)^{\mathrm{a}}$ & $1.985(1.508-2.614)$ & $<0.0001$ & $1.474(1.097-1.980)$ & 0.010 \\
\hline Intrapancreatic neural invasion $(0,1 / 2,3)^{a}$ & $1.655(1.269-2.157)$ & 0.0002 & $1.506(1.145-1.981)$ & 0.003 \\
\hline Histological necrosis (absence/presence) & $2.007(1.531-2.630)$ & $<0.0001$ & $1.853(1.407-2.440)$ & $<\mathbf{0 . 0 0 0 1}$ \\
\hline
\end{tabular}

Univariate and multivariate analyses of prognostic factors associated with disease-specific survival in patients with ductal carcinoma of the pancreas ( $\mathrm{n}=348$ )

\begin{tabular}{|c|c|c|c|c|}
\hline Variables & \multicolumn{2}{|c|}{ Univariate analysis } & \multicolumn{2}{|c|}{ Multivariate analysis } \\
\hline Age $(\geqslant 60$ years $/<60$ years $)$ & $1.054(0.8||-1.37 \mid)$ & 0.692 & & \\
\hline Localisation (pancreas head/body or tail) & $0.829(0.628-1.096)$ & 0.189 & & \\
\hline Tumour size $(\geqslant 30 \mathrm{~mm} /<30 \mathrm{~mm})$ & $1.890(1.37 \mid-2.605)$ & 0.0001 & & \\
\hline Pathologic tumour status $(\mathrm{TI}+\mathrm{T} 2 / \mathrm{T} 3)$ & $6.333(1.572-25.5)$ & 0.009 & & \\
\hline Tumour margin status (negative/positive) & $1.555(1.183-2.043)$ & 0.002 & $1.379(1.038-1.833)$ & 0.027 \\
\hline PL (absence/presence) ${ }^{\mathrm{a}}$ & $1.690(1.267-2.253)$ & 0.0004 & & \\
\hline Lymphatic invasion $(0,1 / 2,3)^{\mathrm{a}}$ & $2.409(1.762-3.293)$ & $<0.0001$ & $1.992(1.440-2.757)$ & $<0.0001$ \\
\hline Venous invasion $(0,1 / 2,3)^{\mathrm{a}}$ & $1.968(1.486-2.607)$ & $<0.0001$ & & \\
\hline Intrapancreatic neural invasion $(0,1 / 2,3)^{\mathrm{a}}$ & $1.709(1.305-2.238)$ & $<0.0001$ & $1.443(1.087-1.915)$ & 0.011 \\
\hline Histological necrosis (absence/presence) & $2.196(1.659-2.905)$ & $<0.0001$ & $2.238(1.686-2.97 \mid)$ & $<\mathbf{0 . 0 0 0 1}$ \\
\hline
\end{tabular}

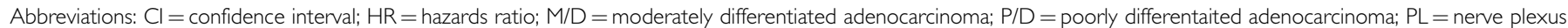
invasion; W/D = well-differentiated adenocarcinoma. ${ }^{a}$ Classified according to the classification of pancreatic carcinoma of Japan Pancreas Society

invasion were combined into two categories (grades 0 and 1 and grades 2 and 3 ) as used in the survival analysis, the $\kappa$-values for lymphatic, venous, and neural invasion were 0.55 (moderate), 0.62 (substantial), and 0.62 (substantial), respectively.

Survival analysis was performed using the data for necrosis identification by the five independent observers, and this yielded similar results, that is, patients having PDC with necrosis showed significantly shorter survival. $P$-values calculated for each of these analyses were $0.0004,0.0005,0.002,0.005,0.006$, and 0.008 for DFS, and $0.0001,0.0004,0.003,0.005,0.005$, and 0.008 for DSS

\section{DISCUSSION}

Pancreatic ductal carcinoma is one of the most aggressive cancers, with almost equivalent incidence and mortality rates. Several histopathological prognostic variables for patients with PDC have been reported (Trede et al, 1990; Yeo et al, 1995; Luttges et al, 2000; Sohn et al, 2000; Lim et al, 2003; Takai et al, 2003; Adsay et al, 2005; Mitsunaga et al, 2005, 2007; Shimada et al, 2006; Schnelldorfer et al, 2008). These are sometimes controversial and complex, and some have problems related to interobserver reproducibility. We need prognostic indicators being simpler, more reproducible, and accurate.
In this study, we reviewed the histopathological findings in 348 cases of PDC in comparison with the corresponding clinicopathological information, and obtained several histopathological prognosticators of both DFS and DSS for patients with PDC by univariate survival analyses employing histological necrosis, tumour size, tumour status, node status, metastatic status, margin status, nerve plexus invasion, and lymphatic, venous, and intrapancreatic neural invasion as variables. Multivariate survival analyses revealed that histological necrosis was an independent predictive factor for both shorter DFS $(P<0.0001)$ and shorter DSS $(P<0.0001)$ of PDC patients (Table 2$)$. In addition, metastatic status, lymphatic invasion, venous invasion, and intrapancreatic neural invasion were factors that were independently predictive of shorter DFS, whereas metastatic status, margin status, lymphatic invasion, and intrapancreatic neural invasion were factors independently predictive of shorter DSS (Table 2). Necrosis was also able to predict patient outcome in populations at stage IIB, into which the majority of resectable PDCs were stratified (Figure 4). Furthermore, the reproducibility of necrosis identification was found to be 'almost perfect' ( $\kappa$-value, 0.87 ) when the 51 slides of PDC were assessed by five independent observers. In contrast, the reproducibility of the systems of grading for lymphatic, venous, and neural invasion was low with low $\kappa$-values. Moreover, these five independent observers all provided the same result, that is, that patients having PDC with necrosis showed 
significantly shorter survival in terms of both DFS and DSS. These findings indicate that histological necrosis is a simple, accurate, and reproducible predictor of postoperative outcome for PDC patients.

Histological necrosis was found in 223 (64.1\%) out of 348 cases of PDC. We defined necrosis as covering both massive necrosis and micronecrosis, the latter being often evident in PDCs, although not noted previously or being hidden as smaller foci (Couvelard et al, 2005; Mitsunaga et al, 2005). The expression of CAIX (Figure 2) and HIF-1 $\alpha$ (data now shown) in and around the necrotic areas was detected immunohistochemically, together with that the presence of necrosis was significantly correlated with expression of CAIX (Table 1), indicating that both patterns of necrosis were closely related to hypoxia. Hasebe's group reported that necrosis was an independent prognostic factor in both DFS and DSS when they investigated histopathological findings in 101 PDC patients (Nakatsura et al, 1997; Mitsunaga et al, 2005). Couvelard et al (2005) reported that necrosis was associated with poorer DSS in univariate analysis, and also showed that necrosis was significantly associated with CAIX expression. Only $30-40 \%$ of PDC cases had necrosis in those studies, whereas $60-80 \%$ of PDCs had hypoxia, as necrosis was defined roughly by these two groups as massive necrosis only.

When massive necrosis and micronecrosis were separated as distinct variables, univariate and multivariate survival analyses demonstrated that both types of necrosis were significantly associated with shorter DFS and DSS (Supplementary Table 2). Massive necrosis and micronecrosis was detected in 27.9 and $43.4 \%$ (overlapped $7.2 \%$ ) of PDCs in our series, respectively. These findings suggest that the presence of necrosis, even when the lesion is small, is closely associated with patient outcome, probably because the presence of necrosis represents a hypoxia-associated aggressive tumour phenotype. In fact, in this series, the presence of necrosis was significantly correlated with a large tumour size, higher $\mathrm{T}$ factor, presence of nodal and distant metastasis, higher TNM stage, more severe venous invasion, and a higher tumour histological grade (Table 1), suggesting that necrosis is positively correlated with tumour growth, invasion, and angiogenesis, that is, an aggressive phenotype.

Hypoxia is a characteristic of invasive cancers that can lead to the development of an aggressive phenotype through a mechanism mediated mainly by HIF- $1 \alpha$, which includes cell immortalisation and dedifferentiation, $\mathrm{pH}$ regulation, autocrine growth/survival, angiogenesis, invasion/metastasis, and resistance to chemotherapy (Semenza, 2006, 2009; Grothey and Galanis, 2009). In fact, the presence of hypoxic foci with expression of CAIX was an independent worse prognostic factor for PDC patients (Supplementary Table 1) that we demonstrated at first time using large series of cases. Recently, HIF-1 targeting therapy and antiangiogenesis therapy have been reported to yield promising anticancer effects (Sessa et al, 2008; Grothey and Galanis, 2009; Semenza, 2009). It is suggested that evaluation of histological necrosis would be useful not only for decision making about postoperative clinical management, but also for stratifying patients for clinical trials aimed at evaluating HIF-1 targeting or antiangiogenesis therapies.

In conclusion, we reviewed the histopathological findings in 348 PDCs for which the presence of necrosis was redefined, and found that histological necrosis was an independent predictor of shorter DFS and DSS for the affected patients. Interobserver reproducibility for the detection of necrosis was assessed as 'almost perfect' when 51 slides of PDC were reviewed by five independent observers. These findings indicate that histological necrosis is a simple, accurate, and reproducible predictor of postoperative outcome in PDC patients.

\section{ACKNOWLEDGEMENTS}

Research support: This work was supported by a Grant-in-Aid for Third Term Comprehensive 10-year Strategy for Cancer Control from the Ministry of Health, Labor and Welfare of Japan (NH and YK), the Program for Promotion of Fundamental Studies in Health Sciences of the National Institute of Biomedical Innovation (YK), and a Grant-in-Aid for Scientific Research from the Ministry of Education, Culture, Sports, Science and Technology of Japan (NH). The authors thank Dr Hidenori Ojima, Dr Yoshihiro Sakamoto, Dr Minoru Esaki, and Dr Satoshi Nara for useful discussion.

Supplementary Information accompanies the paper on British Journal of Cancer website (http://www.nature.com/bjc)

\section{REFERENCES}

Adsay NV, Basturk O, Bonnett M, Kilinc N, Andea AA, Feng J, Che M, Aulicino MR, Levi E, Cheng JD (2005) A proposal for a new and more practical grading scheme for pancreatic ductal adenocarcinoma. Am J Surg Pathol 29: 724-733

Bristow RG, Hill RP (2008) Hypoxia and metabolism. Hypoxia, DNA repair and genetic instability. Nat Rev Cancer 8: 180-192

Center for Cancer Control and Information Services, National Cancer Center Japan (2009) Cancer Statistics in Japan

Chia SK, Wykoff CC, Watson PH, Han C, Leek RD, Pastorek J, Gatter KC, Ratcliffe P, Harris AL (2001) Prognostic significance of a novel hypoxiaregulated marker, carbonic anhydrase IX, in invasive breast carcinoma. J Clin Oncol 19: $3660-3668$

Couvelard A, O'Toole D, Leek R, Turley H, Sauvanet A, Degott C, Ruszniewski P, Belghiti J, Harris AL, Gatter K, Pezzella F (2005) Expression of hypoxia-inducible factors is correlated with the presence of a fibrotic focus and angiogenesis in pancreatic ductal adenocarcinomas. Histopathology 46: 668-676

Fleiss JL (1971) Measuring nominal scale agreement among many rates. Pshycol Bull 76: $378-382$

Gilchrist KW, Gray R, Fowble B, Tormey DC, Taylor SGt (1993) Tumor necrosis is a prognostic predictor for early recurrence and death in lymph node-positive breast cancer: a 10-year follow-up study of 728 Eastern Cooperative Oncology Group patients. J Clin Oncol 11: $1929-1935$
Grothey A, Galanis E (2009) Targeting angiogenesis: progress with antiVEGF treatment with large molecules. Nat Rev Clin Oncol 6: 507-518

Harris AL (2002) Hypoxia - a key regulatory factor in tumour growth. Nat Rev Cancer 2: 38-47

Hruban RH, Pitman MB, Klimstra DS (2007) Ductal adenocarcinoma. In AFIP Atlas of Tumor Pathology. Tumors of the Pancreas, Hruban RH, Pitman MB, Klimstra DS (eds) 4th edn, pp 111-164. ARP Press: Washington, DC

Japan Pancreas Society (2003) Classification of Pancreatic Cancer. 2nd English edn, Kanehara: Tokyo, Japan

Jemal A, Siegel R, Ward E, Hao Y, Xu J, Thun MJ (2009) Cancer statistics, 2009. CA Cancer J Clin 59: 225-249

Kitada T, Seki S, Sakaguchi H, Sawada T, Hirakawa K, Wakasa K (2003) Clinicopathological significance of hypoxia-inducible factor-1alpha expression in human pancreatic carcinoma. Histopathology 43: 550-555

Klöppel G, Adler G, Hruban RH, Kern SE, Longnecker DS, Partanen TJ (2000) Ductal adenocarcinoma of the pancreas. In World Health Organization Classification of Tumours. Pathology \& Genetics. Tumours of the Digestive System, Hamilton SR, Aaltonen LA (eds), pp 221 -230. IARC Press: Lyon

Landis JR, Koch GG (1977) The measurement of observer agreement for categorical data. Biometrics 33: $159-174$

Lim JE, Chien MW, Earle CC (2003) Prognostic factors following curative resection for pancreatic adenocarcinoma: a population-based, linked database analysis of 396 patients. Ann Surg 237: 74-85 
Luttges J, Schemm S, Vogel I, Hedderich J, Kremer B, Klöppel G (2000) The grade of pancreatic ductal carcinoma is an independent prognostic factor and is superior to the immunohistochemical assessment of proliferation. J Pathol 191: $154-161$

Mitsunaga S, Hasebe T, Iwasaki M, Kinoshita T, Ochiai A, Shimizu N (2005) Important prognostic histological parameters for patients with invasive ductal carcinoma of the pancreas. Cancer Sci 96: 858-865

Mitsunaga S, Hasebe T, Kinoshita T, Konishi M, Takahashi S, Gotohda N, Nakagohri T, Ochiai A (2007) Detail histologic analysis of nerve plexus invasion in invasive ductal carcinoma of the pancreas and its prognostic impact. Am J Surg Pathol 31: 1636-1644

Nakatsura T, Hasebe T, Tsubono Y, Ryu M, Kinoshita T, Kawano N, Konishi M, Kosuge T, Kanai Y, Mukai K (1997) Histological prognostic parameters for adenocarcinoma of the pancreatic head. Proposal for a scoring system for prediction of outcome. J Hep Bil Pancr Surg 4: $441-448$

Ord JJ, Agrawal S, Thamboo TP, Roberts I, Campo L, Turley H, Han C, Fawcett DW, Kulkarni RP, Cranston D, Harris AL, Ord JJ, Agrawal S, Thamboo TP, Roberts I, Campo L, Turley H, Han C, Fawcett DW, Kulkarni RP, Cranston D, Harris AL (2007) An investigation into the prognostic significance of necrosis and hypoxia in high grade and invasive bladder cancer. J Urol 178: 677-682

Pastorekova S, Zavadova Z, Kostal M, Babusikova O, Zavada J (1992) A novel quasi-viral agent, $\mathrm{MaTu}$, is a two-component system. Virology 187: $620-626$

Philip PA, Mooney M, Jaffe D, Eckhardt G, Moore M, Meropol N, Emens L, O'Reilly E, Korc M, Ellis L, Benedetti J, Rothenberg M, Willett C, Tempero M, Lowy A, Abbruzzese J, Simeone D, Hingorani S, Berlin J, Tepper J (2009) Consensus report of the national cancer institute clinical trials planning meeting on pancreas cancer treatment. J Clin Oncol 27: $5660-5669$

Schnelldorfer T, Ware AL, Sarr MG, Smyrk TC, Zhang L, Qin R, Gullerud RE, Donohue JH, Nagorney DM, Farnell MB (2008) Long-term survival after pancreatoduodenectomy for pancreatic adenocarcinoma: is cure possible? Ann Surg 247: $456-462$

Semenza GL (2006) Development of novel therapeutic strategies that target HIF-1. Expert Opin Ther Targets 10: 267-280

Semenza GL (2009) HIF-1 inhibitors for cancer therapy: from gene expression to drug discovery. Curr Pharm Des 15: 3839-3843
Sessa C, Guibal A, Del Conte G, Ruegg C (2008) Biomarkers of angiogenesis for the development of antiangiogenic therapies in oncology: tools or decorations? Nat Clin Pract Oncol 5: 378-391

Shimada K, Sakamoto Y, Sano T, Kosuge T, Hiraoka N (2006) Reappraisal of the clinical significance of tumor size in patients with pancreatic ductal carcinoma. Pancreas 33: 233-239

Sohn TA, Yeo CJ, Cameron JL, Koniaris L, Kaushal S, Abrams RA, Sauter PK, Coleman J, Hruban RH, Lillemoe KD (2000) Resected adenocarcinoma of the pancreas-616 patients: results, outcomes, and prognostic indicators. J Gastrointest Surg 4: 567 -579

Sun HC, Qiu ZJ, Liu J, Sun J, Jiang T, Huang KJ, Yao M, Huang C, Sun H-C, Qiu Z-J, Liu J, Sun J, Jiang T, Huang K-J, Yao M, Huang C (2007) Expression of hypoxia-inducible factor-1 alpha and associated proteins in pancreatic ductal adenocarcinoma and their impact on prognosis. Int J Oncol 30: 1359-1367

Takahashi Y, Akishima-Fukasawa Y, Kobayashi N, Sano T, Kosuge T, Nimura Y, Kanai Y, Hiraoka N (2007) Prognostic value of tumor architecture, tumor-associated vascular characteristics, and expression of angiogenic molecules in pancreatic endocrine tumors. Clin Cancer Res 13: $187-196$

Takai S, Satoi S, Toyokawa H, Yanagimoto H, Sugimoto N, Tsuji K, Araki H, Matsui Y, Imamura A, Kwon AH, Kamiyama Y (2003) Clinicopathologic evaluation after resection for ductal adenocarcinoma of the pancreas: a retrospective, single-institution experience. Pancreas 26: 243-249

Trede M, Schwall G, Saeger HD (1990) Survival after pancreatoduodenectomy. 118 consecutive resections without an operative mortality. Ann Surg 211: $447-458$

Vaupel P, Mayer A (2007) Hypoxia in cancer: significance and impact on clinical outcome. Cancer Metastasis Rev 26: 225-239

Wittekind C, Greene FL, Hutter RVP, Klimpfinger M, Sobin LH (2005) UICC TNM Atlas 5th edn, Springer: New York, NY

Yeo CJ, Cameron JL, Lillemoe KD, Sitzmann JV, Hruban RH, Goodman SN, Dooley WC, Coleman J, Pitt HA (1995) Pancreaticoduodenectomy for cancer of the head of the pancreas. 201 patients. Ann Surg 221: 721-731; discussion $731-733$

Zavada J, Zavadova Z, Pastorek J, Biesova Z, Jezek J, Velek J (2000) Human tumour-associated cell adhesion protein MN/CA IX: identification of M75 epitope and of the region mediating cell adhesion. Br J Cancer 82: $1808-1813$ 